Johannes Eurich · Alexander Brink · Jürgen Hädrich

Andreas Langer · Peter Schröder (Hrsg.)

Soziale Institutionen zwischen Markt und Moral 
Forschung Gesellschaft 
Johannes Eurich · Alexander Brink Jürgen Hädrich · Andreas Langer Peter Schröder (Hrsg.)

\section{Soziale Institutionen zwischen Markt und Moral}

Führungs- und Handlungskontexte

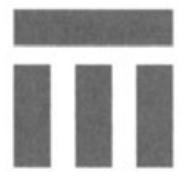


Bibliografische Information Der Deutschen Bibliothek

Die Deutsche Bibliothek verzeichnet diese Publikation in der Deutschen Nationalbibliografie; detaillierte bibliografische Daten sind im Internet über <http://dnb.ddb.de> abrufbar.

Gedruckt mit freundlicher Unterstützung der Evangelischen Altenheimat Stuttgart.

\section{Auflage Oktober 2005}

Alle Rechte vorbehalten

(C) VS Verlag für Sozialwissenschaften/GWV Fachverlage GmbH, Wiesbaden 2005

Lektorat: Monika Mülhausen / Katrin Schmitt

Der VS Verlag für Sozialwissenschaften ist ein Unternehmen von Springer Science+Business Media. www.vs-verlag.de

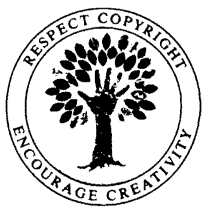

Das Werk einschließlich aller seiner Teile ist urheberrechtlich geschützt. Jede Verwertung außerhalb der engen Grenzen des Urheberrechtsgesetzes ist ohne Zustimmung des Verlags unzulässig und strafbar. Das gilt insbesondere für Vervielfältigungen, Übersetzungen, Mikroverfilmungen und die Einspeicherung und Verarbeitung in elektronischen Systemen.

Die Wiedergabe von Gebrauchsnamen, Handelsnamen, Warenbezeichnungen usw. in diesem Werk berechtigt auch ohne besondere Kennzeichnung nicht zu der Annahme, dass solche Namen im Sinne der Warenzeichen- und Markenschutz-Gesetzgebung als frei zu betrachten wären und daher von jedermann benutzt werden dürften.

Umschlaggestaltung: KünkelLopka Medienentwicklung, Heidelberg

Gedruckt auf säurefreiem und chlorfrei gebleichtem Papier 


\section{Inhaltsverzeichnis}

Johannes Eurich, Alexander Brink, Jürgen Hädrich, Andreas Langer, Peter Schröder

Einleitende Überlegungen zur Reform der sozialen Sicherung zwischen Gerechtigkeit, Solidarität und Ökonomie

\section{Soziale Institutionen zwischen Markt und Moral}

Joachim Wiemeyer

Neoliberalismus und soziale Dienste. Ethik und Ökonomik im Konflikt?

Heinz Schmidt

Marktorientierung und Gerechtigkeit in der Diakonie

Wolfgang Maaser

Gemeinnützige Verbandswirklichkeit im Wandel sozialstaatlicher

Steuerungsmodelle.

Jörg Althammer und Elmar Nass

Gesundheitspolitik zwischen Ressourcenknappheit und sozialethischem Anspruch.

Angela Brand

Die öffentliche Sorge um die Gesundheitsversorgung aller:

Public Health als Vermittler zwischen Ökonomie und Ethik

Eugen Brysch

Mehr Markt schafft Gerechtigkeit. 


\section{Führung in Sozialen Institutionen}

Johannes Eurich und Alexander Brink

Sozialphilosophische und unternehmensethische Aspekte von Führung auf der Grundlage von Anerkennung

Klaus Hildemann

Diakonische Unternehmensführung zwischen

Ethik- und Marktorientierung

Alfred Jäger

Die Funktion der Ethik-Kommission in sozialen Unternehmen.

Andreas Langer

Professionsethik oder Professionsökonomik? Thesen zur Organisation professioneller Dienstleistungen zwischen ökonomischen Instrumenten und weichen Institutionen

\section{Handlungskontexte Sozialer Institutionen zwischen Ethik und Ökonomik}

\section{Karl Kälble}

Die ,Pflege' auf dem Weg zur Profession? Zur neueren Entwicklung der Pflegeberufe vor dem Hintergrund des Wandels und der Ökonomisierung im Gesundheitswesen

Rainer Kretschmer und Gertrud Nass

DRGs im Krankenhausalltag - Ärztliche Entscheidungen im Spagat zwischen knappen Ressourcen und Ethik

\section{Traugott Jähnichen}

Im Spagat zwischen theologischem Anspruch und ökonomischen Zwängen.

Die ,Dienstgemeinschaft' und der arbeitsrechtliche Sonderweg kirchlicher Krankenhäuser vor der Bewährungsprobe 


\section{Vorwort}

Mit dem vorliegenden Herausgeberband wird ein Themenfeld aufgegriffen, das besonders in Zeiten knapper Kassen im Sozial- und Gesundheitssektor ,Konjunktur' hat, obwohl es vor allem im Zusammenhang mit unternehmens- und wirtschaftsethischen Ansätzen bereits breit diskutiert wurde. Die Gestaltung sozialer Institutionen gerät anscheinend mehr und mehr in ein Dilemma zwischen Markt und Moral.

Hier setzen die vorliegenden Beiträge an. Sie beleuchten in einem interdisziplinären Zugang Fragen der Führung und des Handelns in Institutionen des so genannten Dritten Sektors. Das zentrale Anliegen ist dabei nicht, Markt und Moral konkurrierend oder sich ausschließend gegeneinander zu stellen. Vielmehr geht es darum, die berechtigten Ansprüche als notwendige Aufgaben und Herauforderungen zu erkennen, auf die die einzelnen Wissenschaften mit reflektierten und übergreifenden Abwägungen antworten.

Deshalb ist dieser Band auch für Interessierte unterschiedlichster theoretischer und praktischer Herkunft von Interesse. Als erste Buch-Publikation hat dieser Band programmatischen Charakter für die jung gegründete „Forschungsinitiative Ethik und Soziale Institutionen (FESI)“. Die Initiatoren fokussieren Herausforderungen der modernen ,Organisationsgesellschaft' und ihrer institutionellen Landschaft, die sich aus Ansprüchen verschiedener Wertsphären ergeben, wie z.B. dem Spannungsverhältnis zwischen Ökonomik und Ethik. Die Forschungsinitiative ist 2002 aus dem DFG-Graduiertenkolleg „Kriterien der Gerechtigkeit in Ökonomie, Sozialpolitik und Sozialethik“ an der RuhrUniversität Bochum hervorgegangen. Die Wissenschaftler arbeiten in den Bereichen Ökonomie, Diakoniewissenschaft, Sozial- und Gesundheitswissenschaft an der Schnittstelle von Ethik und Ökonomik. Forschungsschwerpunkte liegen auf Fragestellungen des Sozialstaats, des Gesundheitswesens, der Pflegewissenschaften und des professionellen Handelns.

Eine solche Veröffentlichung zu gestalten bereitet Freude, beinhaltet aber auch viel Arbeit. Wir möchten an dieser Stelle allen Autoren für Ihre Beiträge und Mitarbeit danken. Auch sei den verantwortlichen Mitarbeiterinnen auf Ver- 
lagsseite, Frau Monika Mülhausen und Frau Katrin Schmitt, für ihre Anregungen und die Kooperationsbereitschaft gedankt, die uns auch vom VS-Verlag insgesamt entgegengebracht wurde. Verbunden fühlen wir uns auch Herrn Jörg Viebranz und Herrn Moritz Delbrück, die bei der Erstellung der Druckvorlage großen Einsatz gezeigt und uns unschätzbare Dienste geleistet haben. Für Druckkostenzuschüsse bedanken wir uns bei dem Bundesverband evangelische Behindertenhilfe e.V. (www.beb-ev.de), der Universität Bayreuth und der Stiftung Evangelische Altenheimat Stuttgart.

Die Herausgeber

$\begin{array}{lllll}\text { Johannes } & \text { Alexander } & \text { Jürgen } & \text { Andreas } & \text { Peter } \\ \text { Eurich } & \text { Brink } & \text { Hädrich } & \text { Langer } & \text { Schröder } \\ \text { (Heidelberg) } & \text { (Bayreuth) } & \text { (Berlin) } & \text { (Köln) } & \text { (Bielefeld) }\end{array}$

